\title{
An Estimate of the Energy Gap of InN from Measurements of the Fundamental Absorption Edge
}

\author{
P. Trautman*, K. Pakula, A.M. Witowski \\ AND J.M. BARANOWSKI \\ Institute of Experimental Physics, Warsaw University \\ Hoża 69, 00-681 Warszawa, Poland
}

\begin{abstract}
Optical absorption between 0.4 and $4.5 \mathrm{eV}$ of an $\mathrm{InN}$ layer grown by metalorganic vapour phase epitaxy on sapphire was measured at 296 and $12 \mathrm{~K}$. The layer was also characterized by measurements of the Hall effect and of infrared reflectivity in the region of the plasma edge, which determined the concentration, mobility, and effective mass of electrons in the conduction band. The energy gap of InN was estimated to be equal to $0.9 \pm 0.2 \mathrm{eV}$. It was obtained from the spectral position of the fundamental absorption edge. Corrections to the energy gap resulting from the broadening of the fundamental absorption edge, from the Burstein-Moss shift, and from a band-gap shrinkage due to the impurity potential were included.
\end{abstract}

PACS numbers: 73.61.Ey, 78.40.Fy, 78.30.Fs, 78.66.Fd, 81.05.Ea

\section{Introduction}

Indium nitride $(\mathrm{InN})$ is the last classical semiconductor, the energy gap of which is not known. For several years, it was believed that the energy gap $\left(E_{\mathrm{g}}\right)$ of $\mathrm{InN}$ is equal to $1.9 \pm 0.2 \mathrm{eV}$ [1-4]. Since 2002 a number of papers have been published which claim that the $E_{\mathrm{g}}$ of $\mathrm{InN}$ is as low as $0.7 \mathrm{eV}$ [5-11]. This large change of the reported value of the band gap energy of $\mathrm{InN}$ is most likely due to the fact that the early measurements were performed on partially oxidized $\mathrm{InN}$ layers that contained very large concentration of electrons in the conduction band $[12,13]$. The more recent measurements were performed on much purer layers, but still their low crystalline quality did not permit a definitive determination of

*corresponding author; e-mail: Pawel.Trautman@fuw.edu.pl 
the energy gap of InN. In this paper, the energy gap of $\mathrm{InN}$ is estimated from the spectral position of the fundamental absorption edge. The effects on the estimated value of $E_{\mathrm{g}}$ due to broadening of the absorption edge, the Burstein-Moss shift, and a band-gap shrinkage due to the impurity potential are included. This is done in order to estimate the true band gap energy of pure InN from measurements performed on an available strongly $n$-type layer grown by metalorganic vapour phase epitaxy (MOVPE).

\section{Growth of samples and experimental details}

InN layers were grown on sapphire substrates in a MOVPE reactor at the growth temperature of $500^{\circ} \mathrm{C}$. Although these layers were not intentionally doped, one of these layers, which was most suitable for optical absorption measurements, had a free electron concentration equal to $1.05 \times 10^{20} \mathrm{~cm}^{-3}$ and the mobility of only $85 \mathrm{~cm}^{2} /(\mathrm{V} \mathrm{s})$. These values were determined from the Hall effect measurements by the van der Pauw method at a room temperature. The atomic force microscopy and X-ray diffraction measurements indicated that the InN layer had a low crystalline quality. This was mainly due to the fact that $\mathrm{InN}$ could not be grown at a temperature much higher than $500^{\circ} \mathrm{C}$, because $\mathrm{InN}$ rapidly decomposed above this temperature. The tricky technique of growing a buffer layer at low temperature and then growing the main layer at a high temperature, which normally results in GaN layers of good quality [14] has not been developed so far for the growth of InN. The InN layer which is investigated in this paper is strongly $n$-type $\left(n_{\mathrm{H}}=1.05 \times 10^{20} \mathrm{~cm}^{-3}\right)$. It is probably due to the low crystalline quality of the layer and due to the fact that nitrogen was used during InN growth as a carrier gas instead of very pure hydrogen used for the growth of $\mathrm{GaN}$ or $\mathrm{Al}_{x} \mathrm{Ga}_{1-x} \mathrm{~N}$.

Optical transmission spectra were measured with Varian Cary 5 spectrophotometer in the spectral range from 3300 to $270 \mathrm{~nm}(0.4$ to $4.6 \mathrm{eV})$. Below the absorption edge of InN, the intensity of the transmitted light oscillates due to the Fabry-Perot interference in the InN layer. The thickness $(0.53 \mu \mathrm{m})$ of the investigated InN layer was determined from the difference in wavelength between the positions of maximum and minimum of this interference spectrum. It was confirmed that the investigated layer is approximately $0.5 \mu \mathrm{m}$ thick by an inspection of the layer under high-resolution optical microscope. Infrared reflectivity in the region of the plasma edge was measured with Bruker IFS 113v Fourier transform spectrometer.

\section{Experimental results and discussion}

Figure 1 shows the spectrum of optical absorption of the InN layer measured at room temperature between 0.4 and $4.5 \mathrm{eV}$. Below the onset of the absorption edge of InN, the intensity of the transmitted light oscillates due to the Fabry-Perot interference of light in the InN layer. The absorption spectrum of the investigated 


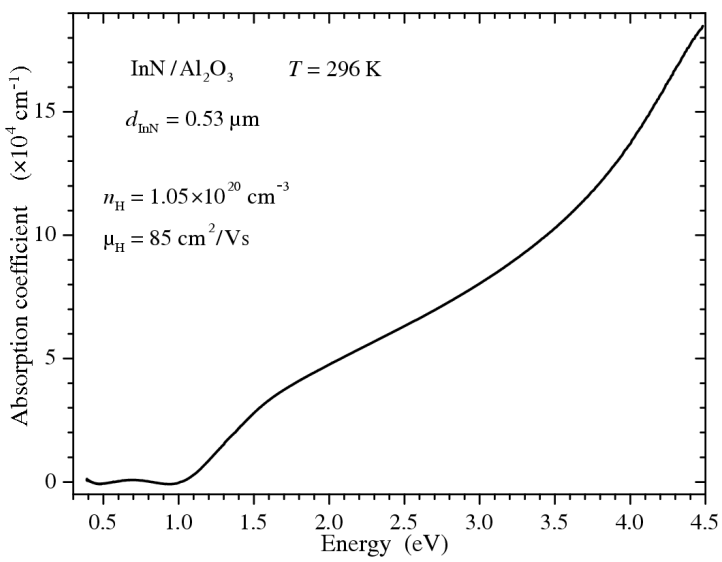

Fig. 1. Absorption spectrum of a $0.53 \mu \mathrm{m}$ thick $\mathrm{InN}$ layer. This layer was grown on sapphire by metalorganic vapour phase epitaxy at the growth temperature equal to $500^{\circ} \mathrm{C}$. The spectrum was measured at room temperature.

InN layer shows two thresholds. The absorption coefficient starts to increase at $1.0 \mathrm{eV}$ and reaches a value of $4 \times 10^{4} \mathrm{~cm}^{-1}$ at $1.6 \mathrm{eV}$, then it increases at a slower rate until a second threshold occurs at $3.4 \mathrm{eV}$ after which the absorption coefficient reaches a value of $2 \times 10^{5} \mathrm{~cm}^{-1}$ at $4.5 \mathrm{eV}$.

Figure 2 shows the effect of reduction of the temperature from room temperature to $12 \mathrm{~K}$. It can be seen from this figure that the cooling of the sample results in marginal, if any, sharpening of the absorption spectrum. The first threshold shifts to a higher energy by $\approx 25 \mathrm{meV}$ after the sample has been cooled from 296 to $12 \mathrm{~K}$. The very small effect of reduction of the temperature and the high value of the absorption coefficient $\left(4 \times 10^{4} \mathrm{~cm}^{-1}\right)$ above the first threshold indicate that it is due to the direct transitions between the valence and conduction bands of InN. The energy gap of pure InN cannot be simply read from the absorption

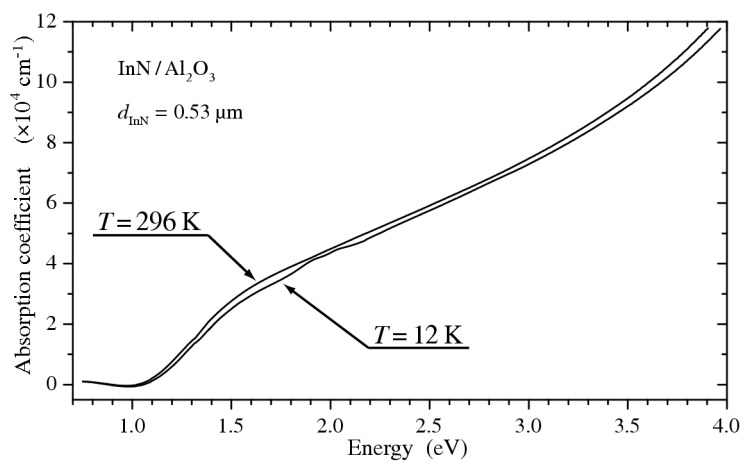

Fig. 2. Absorption spectra of the InN layer measured at 296 and $12 \mathrm{~K}$. The same InN layer was used as that with which the spectrum shown in Fig. 1 was measured. 
spectrum shown in Fig. 1, because this spectrum is distorted from the spectrum of pure and high crystalline quality material by the effects due to the Burstein-Moss shift, broadening, and the impurity potential induced band-gap shrinkage. It is not possible to calculate the magnitudes of these effects precisely, but they can be estimated. First of all, it is necessary to calculate the Fermi level $\left(E_{\mathrm{F}}\right)$ in the investigated sample. It is given by the following formula:

$$
E_{\mathrm{F}}=\frac{h^{2}}{2 m^{*}}\left(\frac{3 n}{8 \pi}\right)^{2 / 3}
$$

Since the concentration $(n)$ of electrons in the conduction band was determined from the measurement of the Hall effect, only the effective mass $m^{*}$ is needed to calculate $E_{\mathrm{F}}$. Curve $(d)$ in Fig. 3 shows the infrared reflectivity of the investigated

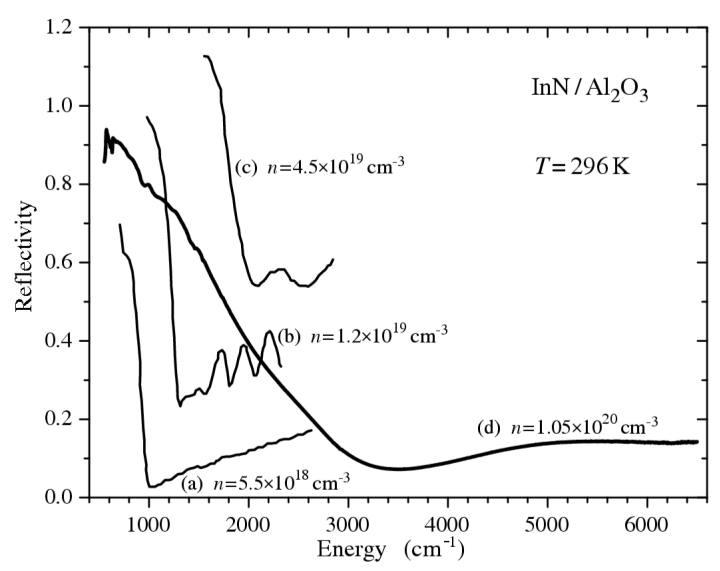

Fig. 3. Infrared reflectivity curves of several InN layers on sapphire measured in the region of the plasma edge. Curves $(a)-(c)$ show for comparison data from Ref. [6] obtained on InN layers grown by molecular beam epitaxy. Curves $(b)$ and $(c)$ are vertically shifted by 0.2 and 0.5 , respectively. Curve $(d)$ shows data obtained on the same InN layer as that used for measurements of absorption spectra shown in Figs. 1 and 2. The plasma edges shown by curves $(a)-(c)$ are substantially sharper than that of curve $(d)$; this is due to much higher mobility $\left(\approx 600 \mathrm{~cm}^{2} /(\mathrm{V} \mathrm{s})\right)$ of electrons in the samples used to measure curves $(a)-(c)$ than that $\left(85 \mathrm{~cm}^{2} /(\mathrm{V} \mathrm{s})\right)$ in sample used for curve $(d)$.

sample in the region of the plasma edge. This curve has a plasma minimum at $\hbar \omega_{\min }=3500 \mathrm{~cm}^{-1}=0.434 \mathrm{eV}$. This frequency is related to the plasma frequency $\omega_{\mathrm{p}}$ and the material parameters by the following equations:

$$
\omega_{\min }=\omega_{\mathrm{p}} \sqrt{\frac{\varepsilon_{\infty}}{\varepsilon_{\infty}-1}} \quad \text { and } \quad \omega_{\mathrm{p}}=\sqrt{\frac{n e^{2}}{\varepsilon_{\infty} \varepsilon_{0} m^{*}}} .
$$

The effective mass of free electrons in the investigated sample is thus equal to 
$m^{*}=0.138 m_{0}$. The value of the high-frequency dielectric constant $\left(\varepsilon_{\infty}\right)$ equal to 6.7 was used after Ref. [15]. The value of $m^{*}$ obtained here for $n=1.05 \times 10^{20} \mathrm{~cm}^{-3}$ is in a good agreement with the dependence of $m^{*}$ on $n$ given in Ref. [6].

Therefore, the Fermi level in the investigated InN layer is equal to

$$
E_{\mathrm{F}}\left(n=1.05 \times 10^{20} \mathrm{~cm}^{-3}\right)=0.59 \mathrm{eV} .
$$

The energy at which the absorption due to the fundamental absorption edge occurs in a crystal of low crystalline quality can be much (by several $100 \mathrm{meV}$ ) lower than the true energy gap of good quality crystal [16]. In the investigated InN crystal the absorption edge starts at $1.0 \mathrm{eV}$, and is smeared over $0.6 \mathrm{eV}$. Thus, it seems that energy gap of InN is equal to $1.3 \mathrm{eV}$ minus the Burstein-Moss shift. This shift is typically smaller than $E_{\mathrm{F}}$ due to a band-gap shrinkage resulting from the impurity potential. Assuming that the band-gap shrinkage due to impurity potential in the investigated $\mathrm{InN}$ sample is equal to $200 \mathrm{meV}$, the band gap of $\mathrm{InN}$ is equal to $0.9 \pm 0.2 \mathrm{eV}$. This value is $0.2 \mathrm{eV}$ higher than the values reported in Refs. [5-11]. This discrepancy is most likely due to the fact that the authors of these publications interpret the energy at which the absorption due to the fundamental absorption edge occurs as the energy gap of InN, thus they completely neglect the effect of broadening of the absorption spectra on the value of the energy gap.

\section{Conclusions}

The analysis of the fundamental absorption edge of $\mathrm{InN}$ grown on sapphire presented in this paper indicates that the energy gap of $\mathrm{InN}$ is equal to $0.9 \pm 0.2 \mathrm{eV}$, which is in reasonable agreement with the values of about $0.7 \mathrm{eV}$ recently reported in the literature [5-11, 17]. A definitive determination of the energy gap of $\mathrm{InN}$ requires investigation of $\mathrm{InN}$ layers of high crystalline quality and with low concentration of free carriers.

\section{References}

[1] H.J. Hovel, J.J. Cuomo, Appl. Phys. Lett. 20, 71 (1972).

[2] K. Osamura, S. Naka, Y. Murakami, J. Appl. Phys. 46, 3432 (1975).

[3] T.L. Tansley, C.P. Foley, J. Appl. Phys. 59, 3241 (1986).

[4] O. Ambacher, J. Phys. D, Appl. Phys. 31, 2653 (1988).

[5] J. Wu, W. Walukiewicz, K.M. Yu, J.W. Ager III, E.E. Haller, H. Lu, W.J. Schaff, Y. Saito, Y. Nanishi, Appl. Phys. Lett. 80, 3967 (2002).

[6] J. Wu, W. Walukiewicz, W. Shan, K.M. Yu, J.W. Ager III, E.E. Haller, H. Lu, W.J. Schaff, Phys. Rev. B 66, 201403 (2002).

[7] V.Yu. Davydov, A.A. Klochikhin, V.V. Emtsev, D.A. Kurdyukov, S.V. Ivanov, V.A. Vekshin, F. Bechstedt, J. Furthmüller, J. Aderhold, J. Graul, A.V. Mudryi, H. Harima, A. Hashimoto, A. Yamamoto, E.E. Haller, Phys. Status Solidi B 234, 787 (2002). 
[8] T. Inushima, M. Higashiwaki, T. Matsui, Phys. Rev. B 68, 235204 (2003).

[9] A.G. Bhuiyan, A. Hashimoto, A. Yamamoto, J. Appl. Phys. 94, 2779 (2003).

[10] I. Vurgaftman, J.R. Meyer, J. Appl. Phys. 94, 3675 (2003).

[11] A. Kasic, E. Valcheva, B. Monemar, H. Lu, W.J. Schaff, Phys. Rev. B 70, 115217 (2004).

[12] M. Yoshimoto, H. Yamamoto, W. Huang, H. Harima, J. Saraie, A. Chayahara, Y. Horino, Appl. Phys. Lett. 83, 3480 (2003).

[13] A.G. Bhuiyan, K. Sugita, K. Kasashima, A. Hashimoto, A. Yamamoto, V.Yu. Davydov, Appl. Phys. Lett. 83, 4788 (2003).

[14] S. Nakamura, Jap. J. Appl. Phys. 30, L1705 (1991).

[15] A. Kasic, M. Schubert, Y. Saito, Y. Nanishi, G. Wagner, Phys. Rev. B 65, 115206 (2002).

[16] P. Trautman, K. Pakula, J.M. Baranowski, Phys. Status Solidi C 2, 1027 (2005).

[17] H. Ahn, C.-H. Shen, C.-L. Wu, S. Gwo, Appl. Phys. Lett. 86, 201905 (2005). 